\title{
Overcoming the Tumor Microenvironment with STING Agonism: Lessons Learned from Viruses
}

\author{
Clavijo-Salomon Maria $\mathrm{A}^{1 *}$, Azevedo Ricardo A², Jorge Salomao $\mathrm{D}^{1,2}$ and Ferreira Adilson $\mathrm{K}^{1,2 *}$ \\ ${ }^{1}$ Laboratory of Tumor Immunology, Department of Immunology, Institute of Biomedical Sciences, University of Sao Paulo, SP - Brazil \\ ${ }^{2}$ Alchemy, Innovation, Research\&Development, Department of Oncology, CIETEC/IPEN, University of Sao Paulo, SP — Brazil
}

Submission: February 11, 2017; Published: March 23, 2017

*Corresponding authors: Clavijo-Salomon Maria A, Department of Immunology, University of Sao Paulo, Brazil, Tel: 55-11-3091-7375; Fax: 55-113091-7224; E-mail: mariasalomon@usp.br

Ferreira Adilson K, Department of Immunology, University of Sao Paulo, Brazil, Tel: 55-11-3091-7375; Fax: 55-11-3091-7224;

E-mail: ferreira-kleber@usp.br

\begin{abstract}
The stimulator of interferon genes (STING) is a cytosolic protein that plays a significant role in immunity to viruses and other intracellular microorganisms. STING acts as a sensor for cytosolic nucleic acids, that after recognition trigger the production of type I interferons and inflammatory chemokines, via IRF3 and STAT6. During antigen presentation by dendritic cells, type I interferons promote the establishment of protective CTL responses. Recent evidence has suggested that STING also play an important role in antitumor immunity. Susceptible to pharmacological activation, the agonism of STING alone or in combination with other therapies showed complete regression of poorly immunogenic tumors, a remarkable efficacy that highlights a new era in cancer immunotherapy. This article reviews recent and promising work on pharmacological activation of STING and provide insight for the rational design of human STING agonists.
\end{abstract}

Keywords: STING; Type I interferons; Antiviral immunity; Anticancer immunotherapy; STING agonism

Abbreviations: APCs: Antigen Presenting Cells; CTLA-4: Cytotoxic T-Lymphocyte Associated Protein 4; DCs: Dendritic Cells; DMXAA: 5,6-Dimethylxanthenone-4-Acetic Acid; HBV: Hepatitis B Virus; HSV-1: Herpes Simplex Virus 1; PD-1: Programmed Cell Death Protein 1; PD-L1: Programmed Death-Ligand 1; STING: Stimulator Of Interferon Genes; TBK1: Tank-Binding Kinase 1; VSV: Vesicular Stomatitis Virus

\section{Introduction}

The stimulator of interferon genes (STING) is a cytosolic protein that in humans is encoded by the TMEM173 gene. It plays a significant role in immunity to viruses and other intracellular microorganisms, acting as a cytosolic sensor for nucleic acids [1,2]. After recognition of DNA in the cytosol, STING re-localizes and binds the TANK-binding kinase 1, TBK1, which phosphorylates the transcription factors IRF3 and STAT6. After activation, IRF3 and STAT6 form dimmers that translocate to the nucleus, inducing the transcription of type I interferons and chemokine genes [3].

Type I interferons are the first line of defense during a virus infection. Having an autocrine and paracrine activity, they allow the infected cells to clear the pathogen while amplifying their effect to surrounding cells [4]. In STING-knockout mice serum, lower titers of IFN- $\alpha$ and IFN- $\beta$ were found after HSV-1 and VSV infection, associated with defective virus clearance and poor survival rates [5].

STING is found in a variety of cells, both immune and nonimmune. Within the immune system, STING is overexpressed in antigen presenting cells (APCs) such as monocytes and dendritic cells (DCs) [6]. When a virus infects an APC, type I interferons produced following STING activation will induce the upregulation of antigen processing and presenting machinery [7]. During antigen presentation by DCs, type I interferons will promote the polarization of Th1 responses and favor crosspresentation to CD8+ T cells [4]. STING-deficient mice infected with baculovirus were unable to produce IFN- $\gamma$ and prime CTLs [7]. Additionally, after immunization with an ovalbumin DNA vaccine, the frequency of CD8+ IFN- $\gamma+$ T cells in the spleen of STING-deficient-mice was significantly decreased [5].

\section{Discussion}

Similar to viruses, STING-deficient mice do not properly promote CTL responses during tumor challenges. Recent work has been shown that DCs can detect tumor DNA through a mechanism mediated by STING. DCs from mice lacking STING exhibited defective CD8+ T cell priming after tumor challenge. Moreover, treatment with anti-CTLA-4 plus anti-PD-1 loses effect in these mice [8]. STING-deficient mice were also resistant 
to the radiation-induced antitumor effect of immunogenic death [9].

Currently, there is a growing interest to manipulate the STING pathway to treat disease. Proof of concept for pharmacologic agonism of STING was published recently. Using in vitro and in vivo models of HBV infection, it was found that macrophages induced a robust type I interferon production which suppressed viral replication in hepatocytes, after treatment with an agonist of mouse STING, the chemotherapeutic drug 5,6-dimethylxanthenone-4-acetic acid (DMXAA) [10]. In murine models of acute myeloid leukemia and melanoma, DMXAA treatment resulted in the expansion of antigen-specific CD8+ T cells with an extraordinary capacity of IFN- $\gamma$ production. Tumor re-challenge and even distant metastases were also rejected $[11,12]$.

Structural differences within murine STING and human STING explain why DMXAA is not able to bind or activate human STING $[3,13]$. Thus, the design and develop of human STING agonists is needed. Recently, synthetic STING agonists demonstrated to enhance antitumor efficacy of a GM-CSF vaccine. Treated mice upregulated the number of infiltrating CD8+ IFN $-\gamma+$ cells within B16 tumors, which concomitantly increased expression of PDL1. Combinations of the vaccine, STING agonist and anti-PD-1 therapy resulted in complete regression of poorly immunogenic CT26 tumors that did not respond to the anti-PD-1 therapy alone or in combination with the vaccine [14]. It is known that $\mathrm{T}$ cell-inflamed tumors are more likely to respond to checkpoint inhibitors, while non-T cell inflamed remain resistant [15]. The activation of the STING pathway in DCs will induce the production of CXCL9 and CXCL10, chemokines that will allow the influx of T cells into the tumors [12]. Since the pre-clinical validation of synthetic STING agonists has achieved remarkable therapeutic power, it is expected that their translation into the clinics turns established chemotherapy/immunotherapyresistant tumors into treatable $[12,14,16]$. Certainly, decreased expression of STING within tumors was recently found to predict poor outcome and prognosis in cancer patients [17].

Finally, an additional and very interesting feature of STING is its recently discovered role in autophagy. During macrophage infection with Mycobacterium tuberculosis, the ubiquitinmediated autophagy of bacterial DNA was mediated by STING [18]. Although, STING co-localizes with many autophagy proteins, it is not clear yet if its agonism will positively or negatively regulate autophagy [3]. Moreover, STING-regulated autophagy might have different outcomes in a cell type-dependent manner. Indeed, STING agonists could have a direct effect on the viability of tumor cells by undetermined mechanisms and small molecules have been shown to induce tumor cell death while activating DCs and macrophages $[11,19]$. This function of STING deserves further investigation.

\section{Conclusion}

A huge advance has been done to understand STING biology in the last years; however, many questions remain open and need to be solved in the bench before translating STING agonism to the bedside. Whether STING agonists will activate IRF3, STAT6 or both signaling pathways, resulting in different cytokine profiles and consequently activating different immunological outcomes; what timing for STING activation will trigger the best antitumor adaptive immune response; how STING roles in immunity and autophagy synergize for an optimal antitumor effect; what kind of tumors will benefit from STING-based immunotherapy and finally, what kind of drugs can increase their efficacy with STING adjuvant agonism to achieve the highest clinical benefit for patients. Based on antiviral immunity, a new and exciting era for anticancer immunotherapies just begins, in which pharmacological agonism takes the scene.

\section{Acknowledgement}

Authors would like to thank Fundação de Amparo à Pesquisa do Estado de São Paulo, FAPESP grants 2013/07273-2, 2014/14267-1 and 2015/18528-7.

\section{References}

1. Barber GN (2015) STING: infection, inflammation and cancer. Nat Rev Immunol 15(12): 760-770.

2. Ishikawa H, Barber GN (2008) STING is an endoplasmic reticulum adaptor that facilitates innate immune signalling. Nature 455(7213): 674-678.

3. Burdette DL, Vance RE (2013) STING and the innate immune response to nucleic acids in the cytosol. Nat Immunol 14(1): 19-26.

4. Welsh RM, Bahl K, Marshall HD, Urban SL (2012) Type 1 Interferons and Antiviral CD8 T-Cell Responses. PLoS Pathog 8(1): e1002352.

5. Ishikawa H, Ma Z, Barber GN (2009) STING regulates intracellular DNA-mediated, type I interferon-dependent innate immunity. Nature 461(7265): 788-792.

6. Retrieved from Genecards. mRNA expression in normal human tissues for TMEM173 Gene.

7. Stubbs SH, Riezu-Boj JI, Mancheño U, Rueda P, Lopez L, et al. (2014) Conventional but not plasmacytoid dendritic cells foster the systemic virus-induced type I IFN response needed for efficient CD8 T cell priming. J Immunol 193(3): 1151-1161.

8. Woo SR, Fuertes MB, Corrales L, Spranger S, Furdyna MJ, et al. (2014) STING-Dependent Cytosolic DNA Sensing Mediates Innate Immune Recognition of Immunogenic Tumors. Immunity 41(5): 830-842.

9. Deng L, Liang H, Xu M, Yang X, Burnette B, et al. (2014) STINGDependent Cytosolic DNA Sensing Promotes Radiation-Induced Type I Interferon-Dependent Antitumor Immunity in Immunogenic Tumors. Immunity 41(5): 843-852.

10. Guo F, Han Y, Zhao X, Wang J, Liu F, et al. (2015) STING Agonists Induce an Innate Antiviral Immune Response against Hepatitis B Virus. Antimicrob Agents Chemother 59(2): 1273-1281.

11. Curran EK, Chen X, Corrales L, Kline J (2014) Activation of the Sting Pathway Enhances Immunity and Improves Survival in a Murine Myeloid Leukemia Model. Blood 124(21): 3759-3759. 
12. Corrales L, Glickman LH, McWhirter SM, Kanne DB, Sivick KE, et al. (2015) Direct Activation of STING in the Tumor Microenvironment Leads to Potent and Systemic Tumor Regression and Immunity. Cell Reports 11(7): 1018-1030.

13. Conlon J, Burdette DL, Sharma S, Bhat N, Thompson M, et al. (2013) Mouse, but not human STING, binds and signals in response to the vascular disrupting agent 5,6-dimethylxanthenone-4-acetic acid. J Immunol. 190(10): 5216-5225

14. Fu J, Kanne DB, Leong M, Glickman LH, McWhirter SM, et al. (2015) STING agonist formulated cancer vaccines can cure established tumors resistant to PD-1 blockade. Sci Transl Med 7(283): 283ra52.

15. Spranger S, Gajewski TF (2016) Tumor-intrinsic oncogene pathways mediating immune avoidance. Oncoimmunology 5(3): e1086862.
16. Bronte V (2014) Tumors STING adaptive antitumor immunity. Immunity 41(5): 679-681.

17. Song S, Peng P, Tang Z, Zhao J, Wu W, et al. (2017) Decreased expression of STING predicts poor prognosis in patients with gastric cancer Scientific Reports 7:39858.

18. Watson RO, Manzanillo PS, Cox JS (2012) Extra cellular M tuberculosis DNA targets bacteria for autophagy by activating the host DNA-sensing pathway. Cell 150(4): 803-815.

19. Ferreira AK, Pasqualoto KFM, Kruyt FAE, Berl FP, Azevedo RA, et al. (2016) BFD-22 a new potential inhibitor of BRAF inhibits the metastasis of B16F10 melanoma cells and simultaneously increased the tumor immunogenicity. Toxicol Appl Pharmacol 295: 56-67.

Your next submission with Juniper Publishers will reach you the below assets

- Quality Editorial service

- Swift Peer Review

- Reprints availability

- E-prints Service

- Manuscript Podcast for convenient understanding

- Global attainment for your research

- Manuscript accessibility in different formats

( Pdf, E-pub, Full Text, Audio)

- Unceasing customer service

Track the below URL for one-step submission https://juniperpublishers.com/online-submission.php 